\title{
Personal Constructs of Anxiety Under the 1,5-Benzodiazepine Derivative Clobazam Related to Trait-Anxiety Levels of the Personality
}

\author{
A. C. Parrott* and R. Kentridge \\ Department of Psychology, University of Leeds, Leeds LS2 9JT, England
}

\begin{abstract}
In an initial interview, 20 volunteer subjects provided 32 personal constructs of anxiety, using Kelly's repertory grid technique. The bipolar ends of the anxiety constructs were then separated by 10 -point rating scales. Subjects could then indicate the degree of anxiety feeling on their own personal constructs. The 20 subjects were divided into high and low trait-anxiety sub-groups, on the basis of Speilberger trait-anxiety (personality) scores. Subjects were administered acute single doses of $20 \mathrm{mg}$ clobazam or matching placebo, in a counterbalanced double-blind design. Anxiety levels on the individual personal constructs of anxiety were measured $2 \mathrm{~h}$ after drug administration. There was a significant ANOVA interaction effect between drug condition and trait-anxiety. Of the ten subjects in the high trait-anxiety sub-group, eight demonstrated reduced levels of personal construct anxiety under clobazam (three significantly). In contrast, of the ten subjects in the low trait-anxiety sub-group, nine demonstrated increased levels of personal construct anxiety (eight significantly). The present results are discussed in relation to previous similar findings. Clobazam and other benzodiazepine derivatives (such as diazepam) are effective anxiolytics with the clinically anxious and with normal volunteers having high trait-anxiety personalities. However, normal subjects with low trait-anxiety personalities often produce paradoxical increases in anxiety feelings.
\end{abstract}

Key words: Anxiety - Trait-anxiety - Personal construct theory - Benzodiazepines - Clobazam

Janke et al. (1979) suggested that the emotionality of the personality was an important modifier of response to drugs. The trait of emotionality was described by Janke et al. (1979) as a broad personality area, which appears in personality inventories under a number of closely related terms, including neuroticism (Eyseneck), emotional lability (Cattell) and traitanxiety (Spielberger). In contrast to these dispositions of the personality, Janke et al. (1979) also described the emotional state at a point in time, which has also been variously labelled, for instance as the level of emotional tension (Janke) or as the level of state-anxiety (Spielberger).

In their review, Janke et al. (1979) suggested that subjects with high emotionality personalities tended to demonstrate

\footnotetext{
* Present Address: Institute of Naval Medicine, Alverstoke, Hampshire PO12 2DL, England

Offprint requests to: A. C. Parrott
}

reduced levels of anxiety feelings in response to benzodiazepines or other anxiolytic agents. In contrast, subjects with low emotionality personalities tended to demonstrate paradoxical increases in self-reported feelings of anxiety in response to benzodiazepine or other anxiolytic medications. Barrett and DiMascio (1966) demonstrated significantly different changes in measures of state-anxiety between subject groups with high and low anxiety personalities in response to low doses of diazepam and chlordiazepoxide (CDP). Also, Malpas et al. (1974) reported different psychological performance test assessments for anxious patients, when compared to non-anxious subjects, in their responses to various benzodiazepine derivatives. Despite these and other similar significant findings, in some of the studies in the Janke review significant effects of the emotionality of the personality were not found, while in other studies it was reported with certain drug conditions or dose levels, but not with others (Janke et al. 1979). Differences in subject groups, drug and dose regimens and testing conditions will all have contributed to the variation in findings. However, a further problem within these studies is in defining the anxiety-feeling state, since it is important to describe feelings of anxiety for each individual subject as accurately as possible.

Kelly (1955) proposed the theory of personal constructs by which each individual construes, or understands, their world. Kelly also described the repertory grid technique as a means of delineating each individual's personal constructs. The repertory grid technique has been successfully used to elicit personal constructs pertaining to a wide range of areas (Bannister and Fransella 1980; Stewart and Stewart 1981). In the present study, constructs concerned specifically with anxiety feelings were elicited from each subject. Levels of anxiety, as indicated on the individual subjects' personal constructs, were then measured after the administration of either placebo or clobazam. The repertory grid technique therefore allows each individual to describe their own anxiety feelings. This technique has some advantages over the use of questionnaires based upon group data, where the questions may differ in their relevance or appropriateness for different subjects.

Clobazam, a 1,5-benzodiazepine derivative, has been shown to be an effective anxiolytic, equal in efficacy to diazepam with the clinically anxious (Brodgen et al. 1980; Koeppen 1981). However, in a study with normal volunteer subjects (Steiner-Chaskel and Lader 1981), paradoxical increases in anxiety were found with both clobazam and diazepam. Clyde (1981) also demonstrated significantly different changes in state-anxiety, with high and low traitanxiety sub-groups, under two dose levels of clobazam. 
Clobazam has also been shown to produce changesin performance test assessments with correlate significantly with personality measures of emotionality and anxiety (Parrott and Hindmarch 1975, 1977, 1978; Clyde 1981; Parrott 1982).

The present study was intended to investigate changes in anxiety feelings under clobazam, as indicated by personal constructs of anxiety, in normal volunteer subjects divided into low and high trait-anxiety sub-groups. Performance test assessments were not given in the present study. Janke et al. (1979) suggested that normal (low trait-anxiety) subjects may be perceiving drug-induced impairments on the performance assessments they are required to undertake in many psychopharmacological studies. The perception of impaired performance is then hypothesised to lead to the paradoxical increases in state-anxiety. The present study would indicate whether paradoxical increases in anxiety still occurred in situations where concomitant performance assessments were not being measured.

\section{Materials and Methods}

Subjects completed the Speilberger trait-anxiety form (STAI $x-2)$ in accordance with the suggested procedures (Speilberger et al. 1968). These scores were later and used to classify subjects into either the low or high trait-anxiety subgroups ( $N=10$ per sub-groups, with a cut-off score of 40 ).

Personal Constructs of Anxiety. Personal constructs were elicited using the procedure recommended by Stewart and Stewart (1981) for normal subjects, since Kelly's original description was clinically based (Kelly 1955). The subject was asked to describe six situations which made them anxious (termed the six basic elements $\mathrm{E} 1-\mathrm{E} 6$ ). The subject was then presented with three elements (e.g. E1, E2, E3) and asked to state how two of these were similar to each other in the way they made them feel anxious, but different from the third (e.g. 'feeling cold'): the subject was then asked to indicate the opposite (e.g. 'feeling sociable'). This constitutes the first bipolar construct ( $\mathrm{C} 1$, feeling cold - feeling sociable). A second bipolar construct was elicited (C2) in a similar fashion using the same three elements. Then a different set of three elements were presented (e.g. E1, E3, E5), and two further bipolar constructs elicited (C3, C4). Eight constructs were elicited this way, representing the non-laddered constructs obtained from anxiety situations.

The next stage was to elicit eight laddered constructs (Stewart and Stewart 1981). Each subject was presented with the anxious pole of one of the first eight constructs $(\mathrm{C} 1-\mathrm{C} 8)$ and asked 'What is it about this (e.g. 'I upset may parents') that makes you feel anxious?: The answer (e.g. 'I want to be wanted') and its opposite constitute the first bipolar laddered construct (C9). Eight laddered constructs (C9-C16) were obtained by laddering each of the initial constructs $(\mathrm{C} 1-\mathrm{C} 8)$.

Then the subject was asked to describe six situations where they felt 'non-anxious' (i.e. six non-anxious elements). Using these elements, 16 further bipolar constructs were elicited. Subjects initially indicated how two elements were similar in making them feel non-anxious, then indicated the opposite, which represented the anxious pole of the construct. Eight non-laddered and eight laddered constructs were derived from the non-anxious elements.

Overall, 32 personal constructs were elicited from each individual subject during the initial interview (16 constructs laddered, 16 non-laddered), half derived originally from the anxious elements and half derived from the non-anxious elements.

Personal Constructs of Anxiety: Questionnaire. The 32 personal constructs of anxiety were then formulated into a questionnaire by separating the bipolar opposites of each construct by a 10-point linear rating scale. Constructs were listed in a random order, with the poles of the constructs systematically reversed. The subject circled one of the points of the scale to indicate their anxiety feeling at that point in time. After completion, the questions were scored from 1 (minimum anxiety score) to 10 (maximum anxiety score)

Drug Conditions. Clobazam (20 mg) and placebo, in matching capsules, were given with the order of drug administration counterbalanced. Double-blind testing occurred $2 \mathrm{~h}$ after an acute single dose, since Rupp et al. (1979) indicated peak clobazam serum levels $1-4 \mathrm{~h}$ after an acute single dose.

Subjects and Procedure. The 20 subjects ( 9 male, 11 female; $18-31$ years of age) were in normal physical and mental health, with no history of cardiac, hepatic, renal or mental disorder and were not being prescribed any medication (apart, possibly, from oral contraceptives). Subjects agreed not to drive motor vehicles, operate machinery or consume alcohol on the test days.

During an initial interview subjects completed the Speilberger trait-anxiety questionnaire and provided their 32 personal constructs of anxiety. Two testing sessions were then given at weekly intervals, when the subjects were administered the drug conditions and completed their personal constructs of anxiety questionnaires. Testing occurred at the same time each week. The personal construct procedure for measuring anxiety has been described in more details elsewhere (Kentridge and Parrott, unpublished data).

\section{Results}

The group data for the personal constructs of anxiety were analysed by three-way analysis of variance (ANOVA) for the effects of trait-anxiety (low - high Speilberger trait-anxiety sub-group), laddering of constructs (laddered-non-laddered) and drug condition (clobazam-placebo). The results of this analysis are presented in Table 1. There was a significant main effect for trait-anxiety $(P<0.01)$ and a significant interaction between trait-anxiety and drug condition $(P<0.01)$.

The group mean personal construct anxiety scores are presented in Table 2, where the scores for the laddered and non-laddered constructs were combined since the effect of laddering was non-significant. The significant ANOVA main effect for trait-anxiety reflects the lower personal construct anxiety score for the low trait-anxiety group (85.5) compared with the high trait-anxiety group (104.1). This was expected, since individuals with high trait-anxiety personalities tend to exhibit higher levels of anxiety-feeling states (Speilberger et al. 1968).

The significant ANOVA interaction between drug condition and trait-anxiety reflects the lower placebo (81.0) than clobazam (90.0) personal construct anxiety scores with the low trait-anxiety sub-group, in comparison with the lower clobazam (102.2) than placebo (106.0) personal construct 
Table 1. Personal constructs of anxiety: summary of the three-way analysis of variance findings

\begin{tabular}{lll}
\hline Source of variation & $F$ ratio & $\begin{array}{l}\text { Significance } \\
\text { level }\end{array}$ \\
\hline $\begin{array}{l}\text { Main effects } \\
\quad \text { Trait anxiety (low/high) }\end{array}$ & 8.11 & $P<0.01$ \\
$\quad \begin{array}{l}\text { Drug condition (clobazam-placebo) } \\
\quad 1.18\end{array}$ & - \\
$\quad \begin{array}{l}\text { Laddering of construct (laddered- } \\
\text { non-laddered) }\end{array}$ & 0.59 & - \\
$\begin{array}{l}\text { Interactions } \\
\quad \text { Trait anxiety- } \times \text {-drug condition }\end{array}$ & 7.83 & $P<0.01$ \\
$\quad \begin{array}{l}\text { Trait anxiety- } \times \text {-laddering } \\
\text { Drug condition- } \times \text {-laddering } \\
\text { Trait anxiety- } \times \text {-drug condition- } \times-\end{array}$ & 0.03 & - \\
$\quad$ laddering & 0.45 & - \\
\hline
\end{tabular}

Table 2. Personal constructs of anxiety: group mean scores. Lower scores indicate lower anxiety-feeling states

\begin{tabular}{lccc}
\hline Trait-anxiety sub-group & \multicolumn{3}{l}{ Drug condition } \\
\cline { 2 - 4 } & Placebo & Clobazam & Average \\
\hline Low trait-anxiety & 81.0 & 90.0 & 85.5 \\
High trait-anxiety & 106.0 & 102.0 & 104.1 \\
\hline
\end{tabular}

Table 3. Personal constructs of anxiety. Numbers of subjects demonstrating increases or decreases in anxiety under clobazam (compared to placebo) and significance levels for these differences

\begin{tabular}{|c|c|c|c|c|}
\hline \multirow[t]{2}{*}{$\begin{array}{l}\text { Trait-anxiety } \\
\text { sub-group }\end{array}$} & \multicolumn{2}{|c|}{$\begin{array}{l}\text { Number of subjects } \\
\text { demonstrating anxiety } \\
\text { increase under clobazam } \\
\text { (compared to placebo) }\end{array}$} & \multicolumn{2}{|c|}{$\begin{array}{l}\text { Number of subjects } \\
\text { demonstrating anxiety } \\
\text { decrease under clobazan } \\
\text { (compared to placebo) }\end{array}$} \\
\hline & Significant & $\begin{array}{l}\text { Not } \\
\text { significant }\end{array}$ & $\begin{array}{l}\text { Not } \\
\text { significant }\end{array}$ & Significant \\
\hline $\begin{array}{l}\text { Low trait-anxiety } \\
\qquad(N=10)\end{array}$ & 8 & 1 & 1 & 0 \\
\hline $\begin{array}{l}\text { High trait-anxiety } \\
\quad(N=10)\end{array}$ & 1 & 1 & 5 & 3 \\
\hline
\end{tabular}

anxiety scores demonstrated by the high trait-anxiety subgrop (Table 2).

The personal construct anxiety scores for each subject were also analysed individually for the significance for drug effects by means of paired Student's $t$-tests. The results of these analyses are presented in Table 3 . It can be seen that most of the high trait-anxiety subjects demonstrated reductions in personal construct anxiety levels under clobazam (three significant reductions, five non-significant reductions). In contrast, most of the low trait-anxiety subjects demonstrated increases in personal construct anxiety levels (eight significant increases, one non-significant increase).

\section{Discussion}

In the studies reviewed by Janke et al. (1979), long-term personality characteristics (such as neuroticism, emotional lability) were contrasted with short-term characteristics such as mood states of particular points in time. With regard to anxiety, Speilberger et al. (1968), have empirically demonstrated the important distinction between trait-anxiety as an enduring personality characteristic and state-anxiety, which indicates the level of anxiety feelings at the particular point in time. In relation to drug response, Janke et al. (1979) concluded that people with high emotionality (neuroticism, trait-anxiety) personality scores 'tend to react with a reduction of symptoms connected with emotional tension', while people with low emotionality (neuroticism, traitanxiety) personality scores, tend to respond with 'paradoxical increases in self-reported emotional tension'. The results of the present investigation confirmed these conclusions. The hypothesised interaction between trait-anxiety and change in anxiety-feeling state under clobazam was empirically confirmed by the significant ANOVA interaction between traitanxiety and drug condition (Table 1). The separate analyses of each individuals' constructs under the two drugs conditions further confirmed the importance of trait-anxiety. Under clobazam, subjects with high trait-anxiety personalities generally demonstrated reduced feelings of anxiety. In contrast, subjects with low trait-anxiety personalities generally demonstrated increased feelings of anxiety (Table 3).

Previous investigations have found significant interactions between personality measures of emotionality and druginduced changes in mood state. Barrett and DiMascio (1966) reported that, with $15 \mathrm{mg} C D P$, high-anxiety subjects demonstrated a significant reduction in anxiety, while low-anxiety subjects demonstrated a significant increase in anxiety levels: similar findings were also found with low-dose $(6 \mathrm{mg})$ diazepam. Clyde (1981) reported a significant effect of traitanxiety upon changes in state-anxiety under two dose levels (10 and $20 \mathrm{mg}$ ) of clobazam. The high trait-anxiety subjects demonstrated significant reductions in state-anxiety under clobazam, while the low trait-anxiety subjects demonstrated little change in state-anxiety. Similar significant differences were found for anxiety mood states when measured on a visual analogue scale (Clyde 1981). Janke (1964) with meprobamate, and Schafer-Plog (1968) with fluphenazine also reported significant differences between subjects differing in personality measures of emotionality. However, in some studies, similar significant effects have not been reported; these include Richter and Hobi (1975) with bromazepam, Clyde (1981) with nitrazepam and Barrett and DiMascio (1966) with higher dose levels of diazepam $(15 \mathrm{mg})$, CDP $(30 \mathrm{mg})$ and meprobamate $(1200 \mathrm{mg})$. The above studies have all been concerned with the personality characteristics of volunteer subjects. In studies involving clinically anxious patients, state-anxiety levels are widely reduced by clobazam and other benzodiazepines (Brodgen et al. 1980; Koeppen 1981). However, Rickels (1978), in a review of the effectiveness of anxiolytic agents, suggested that 'the more anxiety a patients has, the better chance he usually has of improving with anxiolytic drug therapy'.

Janke et al. (1979) suggested that performance level changes under psychoactive drugs may also be closely related to the emotionality of the personality. With reference to clobazam, significant correlations between different personality measures (EPI neuroticism scale, MHQ anxiety subscale), and different performance measures (choice reaction time, card sorting, critical flicker-fusion) have been empirically demonstrated (Parrott and Hindmarch 1975, 1977, 
1978; Clyde 1981; Parrott 1982). High trait-anxiety subjects tended to produce significantly better performance changes than the low trait-anxiety subjects under clobazam. Janke et al. (1979) suggested that a possible cause of the paradoxical increase in anxious mood with the low trait-anxiety subjects was that they perceived the drug treatment to interfere with their test performance, thus they felt more anxious. In the present study, no performance tests were given, yet the paradoxical increase in anxiety was still found. Similarly, Barrett and DiMascio (1966) did not administer performance tests, yet found significant mood impairments with $15 \mathrm{mg}$ CDP given to the low personality-anxiety group). Therefore mood impairments may occur even when concomitant performance assessments are not given. However, the subjects may still be perceiving impaired 'performance' in their functioning without undertaking laboratory test assessments.

The feeling of 'loss of control' has also been suggested as an explanation for the paradoxical increase in anxiety (Janke et al. 1979). This hypothetical explanation can also be related to a neurophysiological model of anxiety and benzodiazepine action (Gray 1981a, b). According to Gray's model, the septal-hippocampal system functions as a 'stimulus comparator' system where the importance and relevance of incoming stimuli are assessed. Individuals with high-anxiety personalities would have stimulus comparator systems which respond to a comparatively large number of stimuli as threatening or anxiety provoking, whereas individuals with low-anxiety personalities would process less stimuli as anxiety provoking. According to the model, benzodiazepines and other anxiolytic agents act by reducing the activity of the stimulus comparator system. Therefore, benzodiazepines given to subjects with high-anxiety personalities will lead to less stimuli being seen as anxiety provoking (i.e. a reduction in anxiety feelings). However, benzodiazepines given to subjects with low-anxiety personalities will lead to impairments in stimulus events, as hypothesised by Janke et al. (1979). previously functioning at stable (non-neurotic) levels. This may therefore lead to the feeling of a 'lack of control' over stimulus events, which as hypothesised by Janke et al. (1979). This neurophysiological explanation is, however, only tentative and other models might also explain the findings. The explanation could, however, be empirically investigated by further use of the personal construct procedure. Constructs specifically referring to 'control over stimulus events' could be elicited and measured under drug and placebo conditions. It would then be predicted that feelings of 'lack of control' would show a decrement with low trait-anxiety subjects only. It would, however, be important to also include contructs relating neither to 'anxiety' nor to 'feelings of control' in order to assess whether perceived decrements were specific to the hypothesised areas or whether they occurred more generally with the low trait-anxiety subjects.

Paradoxical responses to benzodiazepines and other anxiolytic agents have been reported in the clinic, where hositility reactions and increases in anxiety have been noted (Byck 1975; DiMascio 1973). Byck (1975) suggested that some high trait-anxiety subjects may suffer from the feeling of lack of control and impairments in their need to master their situation, an explanation which has already been described for low trait-anxiety subjects.

In laboratory psychopharmacological investigations, normal volunteer subjects with unselected personality profiles are frequently used (McNair 1973; Parrott and Hindmarch 1977, 1978; Steiner-Chaskel and Lader 1981; Thompson and
Trimble 1981). In a review of studies concerned with the effects of anti-anxiety agents, McNair (1973) concluded that the findings were inconsistent and frequently conflicting. Although McNair (1973) described several factors contributing to this variation, an important factor, not described in his review, is the variation in personality profiles of different subject groups. Groups with a (random) preponderance of low-emotionality subjects would tend to produce findings different from studies where the group contained a preponderance of high-emotionality subjects. Therefore, it should be suggested that, in studies of anxiolytic agents using normal volunteer subjects, only those with high-emotionality scores should be used. This procedure is already in operation in some laboratories (Biehl 1979). Although a high cut-off point may be used [Biehl (1979), top 15\% neuroticism scores; Clyde (1981), top 25\% trait- and state-anxiety scores], a lower cut-off including the top $50 \%$ of normal subjects may be equally effective in obtaining findings which can generalise to the clinically anxious (Parrott 1982).

The present investigation confirmed the practical utility of personal construct theory. The theoretical strength of the theory is that it is based upon the individuals describing their own personal knowledge and perceptions, and the repertory grid technique then allows these to be formalised and measured. This procedure has benefits over techniques based upon group data where, in the construction of the questionnaire, individual or idiosyncratic responses may become discarded in favour of responses found more frequently with in the total group (Kentridge and Parrott, data in preparation). The repertory grid technique is, however, timeconsuming to administer in comparison with other procedures (visual analogue scales or group questionnaires), although it does elicit far more detailed information. Personal construct theory has a wide range of applicability (Bannister and Fransella 1980; Stewart and Stewart 1981). In future studies, it would therefore be useful to include personal constructs pertaining to a wider range of areas (e.g. anxiety feelings together with self-perceptions of performance ability and feelings of control over stimulus events), and investigate how they are affected by benzodiazepines and other psychoactive medications.

Acknowledgements. The authors would like to thank Hoechst (UK) for providing the matching drug conditions.

\section{References}

Bannister D, Fransella F (1980) Inquiring man: The psychology of personal constructs. Penguin, London

Barrett JE, and Di Mascio A (1966) Comparative effects on anxiety of the minor tranquilisers in high and low anxious student volunteers. Dis Nerv Syst 27:483-486

Bieh1 B (1979) Studies of clobazam and car driving. Br J Clin Pharmacol (Suppl) 7:85-90

Brogden RN, Heel RC, Speight RM, Avery GS (1980) Clobazam: A review of its pharmacological properties and therapeutic use in anxiety. Drugs 20:161-178

Byck R (1975) Drugs and the treatment of psychiatric disorders. In: Groodman LS, Gilman A (eds) The pharmacological basis of therapeutics. Macmillan, New York, pp 152-200

Clyde C (1981) The influence of personality on response to low doses of benzodiazepines. R. Soc Med Int Symp Ser 43:75-86

DiMascio A (1973) The effects of benzodiazepines on aggression: Reduced or increased? In: Garattini S, Mussini E, Randall O (eds) The benzodiazepines. Raven, New York, pp 433-440 
Gray JA (1981a) Anxiety as a paradigm case of emotion. Br Med Bull $37: 193-197$

Gray JA (1981b) The neuropsychology of anxiety: An enquiry into the functions of the sepo-hippocampal system. Oxford University Press, Oxford

Janke W (1964) Experimentelle Untersuchungen zur Abhängigkeit der Wirkung psychotroper Substanzen von Persönlichkeitsmerkmalen. Akademische Verlagsgesellschaft, Frankfurt

Janke W, Debus G, Longo N (1979) Differential psychopharmacology of tranquilizing and sedating drugs. In: Ban TA et al. (eds) Modern problems in pharmacopsychiatry, vol 14. Karger, Basel, pp 13-98

Kelly GA (1955) The psychology of personal constructs, vols 1 and 2. Norton, New York

Koeppen D (1981) Clinical experience with clobazam 1968-1981. R Soc Med Int Symp Ser 43:193-198

McNair DM (1973) Antianxiety drugs and human performance. Arch Gen Psychiatry 29:611-617

Malpas A, Legg NJ, Scott DF (1974) Effects of hypnotics on anxious patients. Br J Psychiatry 124:482-484

Parrott AC (1982) Critical flicker-fusion thresholds and their relationship to other measures of alertness. Pharmacopsychiatria (Suppl) 15: $39-43$

Parrott AC, Hindmarch I (1975) Clobazam: A 1,5-benzodiazepine derivative - effects on anxiety, arousal and performance compared with those of CNS stimulants and tranquilisers. IRCS Med Sci 3:177

Parrott AC, Hindmarch I (1977) Comparative effects of three benzodiazepine derivatives clobazam, nitrazepam and flurazepam upon psychomotor performance under different reinforcement conditions. IRCS Med Sci 5:166
Parrott AC, Hindmarch I (1978) Clobazam: A 1,5 benzodiazepine derivative - effects on human psychomotor performance under different levels of task reinforcement. Arch In Pharmacodyn Ther 223:261-268

Richter R, Hobi V (1975) Die persönlichkeitsspezifische Wirkung eines Tranquilizers. Arzneim Forsch 25:26-28

Rickels K (1978) Use of antianxiety agents in anxious outpatients. Psychopharmacology 58:1-17

Rupp W, Badian M, Christ O, Hajdu P, Kulkarni RD, Taeuber K, Uihlein M, Bender R, Vanderbeke O (1979) Pharmacokinetics of single and multiple doses of clobazam in humans. Br J Clin Pharmacol (Suppl) 7:51-58

Schafer-Plog U (1968) Die Wirkung von Fluphenazin auf das Testverhalten von Versuchspersonen unter Berücksichtigung der vegetativen Labilität und Einflusses des Versuchsleiters. Arzneim Forsch 18:443-447

Speilberger CD, Gorsuch R, Lusheine RG (1968) Manual for the statetrait anxiety inventory. Florida State University, Florida

Steiner-Chaskel N, Lader MH (1981) Effects of single doses of clobazam and diazepam on psychological functions in normal subjects. R Soc Med Int Symp Ser $43: 23-32$

Stewart V, Stewart A (1981) Business applications of the repertory grid. McGraw Hill, London

Thompson PJ, Trimble MR (1981) Clobazam and cognitive functions: effects in healthy volunteers. R Soc Med Int Symp Ser 43:33-38

Received May 25, 1982 